\title{
Arterial Wall Imaging in Angiographically Occult Sponta- neous Subarachnoid Hemorrhage : New Insight into the Usual Suspect
}

\author{
Wonki Yoon, Jang Hun Kim, ${ }^{2}$ Haewon Roh, ${ }^{3}$ Taek-Hyun Kwon ${ }^{1}$ \\ Department of Neurosurgery, Korea University Guro Hospital, Korea University College of Medicine, Seoul, Korea \\ Department of Neurosurgery, ${ }^{2}$ Seoul National University Bundang Hospital, Seoul National University College of Medicine, Seongnam, Korea \\ Department of Neurosurgery, ${ }^{3}$ Armed Forces Capital Hospital, Seongnam, Korea
}

Objective : The etiology of angiographically occult spontaneous subarachnoid hemorrhage (AOsSAH) is unclear. Threedimensional (3D) high-resolution vessel wall magnetic resonance imaging (HVM) might be useful in detecting the hidden arterial wall angiopathy in patients with AOsSAH. We aimed to demonstrate the feasibility of HVM for detecting the arterial cause of AOsSAH.

Methods : Patients, who were diagnosed with AOSSAH in the first evaluations and underwent HVM, were enrolled. Their clinical and radiologic data were retrospectively reviewed. Especially, focal enhancement of arterial wall on HVM and repetitive catheterized angiograms were precisely compared.

Results : Among 251 patients with spontaneous $\mathrm{SAH}, 22$ patients were diagnosed with AOsSAH in the first evaluations (8.76\%). After excluding three patients who did not undergo 3D-HVM, 19 patients were enrolled and classified as convexal $(n=2)$ or perimesencephalic $(n=4)$, and diffuse $(n=13)$ groups. In convexal and perimesencephalic groups, no focal enhancement on HVM and no positive findings on repetitive angiography were noted. In diffuse group, 10 patients showed focal enhancement of arterial wall on HVM $(10 / 13,76.9 \%)$. Repeated angiography with 3D reconstruction revealed four patients of angiographically positive causative arteriopathy and possible lesion in one case in the concordant location of intramural enhancement on 3D-HVM (5/10, 50\%). Three of them were treated with endovascular stent insertion. All patients, except one, recovered with good clinical outcome (3-month modified Rankin score, 0 and 1 ).

Conclusion : 3D-HVM was useful in detecting hidden true arteriopathy in AOsSAH. It may provide new insights into the etiologic investigation of AOsSAH by proving information about the arterial wall status.

Key Words : Angiography · Aneurysm · Magnetic resonance imaging, 3D high resolution vessel wall · Subarachnoid hemorrhage.

- Received : May 20, $2021 \cdot$ Revised : August 19, $2021 \cdot$ Accepted : October 6, 2021

- Address for reprints : Taek-Hyun Kwon

Department of Neurosurgery, Korea University Guro Hospital, Korea University College of Medicine, 148 Gurodong-ro, Guro-gu, Seoul 08308, Korea Tel : +82-2-2626-1178, Fax : +82-2-863-1864, E-mail : ns806@kumc.or.kr, ORCID : https://orcid.org/0000-0002-8428-6952 


\section{INTRODUCTION}

Aneurysmal rupture is the most common cause of spontaneous subarachnoid hemorrhage (SAH). Other causes include dissection, arteriovenous fistula, arteriovenous malformation, and other cerebral arteriopathies ${ }^{17)}$. These causes can be revealed by angiographic evaluation. However, the reported incidence of angiographically occult spontaneous SAH (AOsSAH) is approximately $20 \%{ }^{3,10)}$. For investigating the source of AOsSAH, two or more successive angiographic follow-up evaluations are routinely performed. However, their detection rate is as low as $7-15 \%^{11,12,28)}$. Spinal and brain magnetic resonance imaging (MRI) also has a very low detection rate ${ }^{8,24,27)}$.
Several vasculopathies such as deep venous system anomalies, vasculitis, and perforator aneurysm rupture are hypothesized as the possible causes of AOsSAH ${ }^{4,18,25)}$. However, the definitive pathological cause is unclear.

According to the quantity and the location of hemorrhage, AOsSAH can be classified into three types : perimesencephalic, convexal, and diffuse $\mathrm{e}^{12,15,17,23)}$. The clinical course of AOs$\mathrm{SAH}$ is benign, especially in the convexal and perimesencephalic types; however, the diffuse type shows a clinical course resembling aneurysmal SAH. Approximately 20\% diffuse AOsSAH cases exhibit a complicated course with delayed cerebral ischemia, hydrocephalus, and even rebleeding ${ }^{3,13,15)}$. These features and the amount of SAH in diffuse AOsSAH
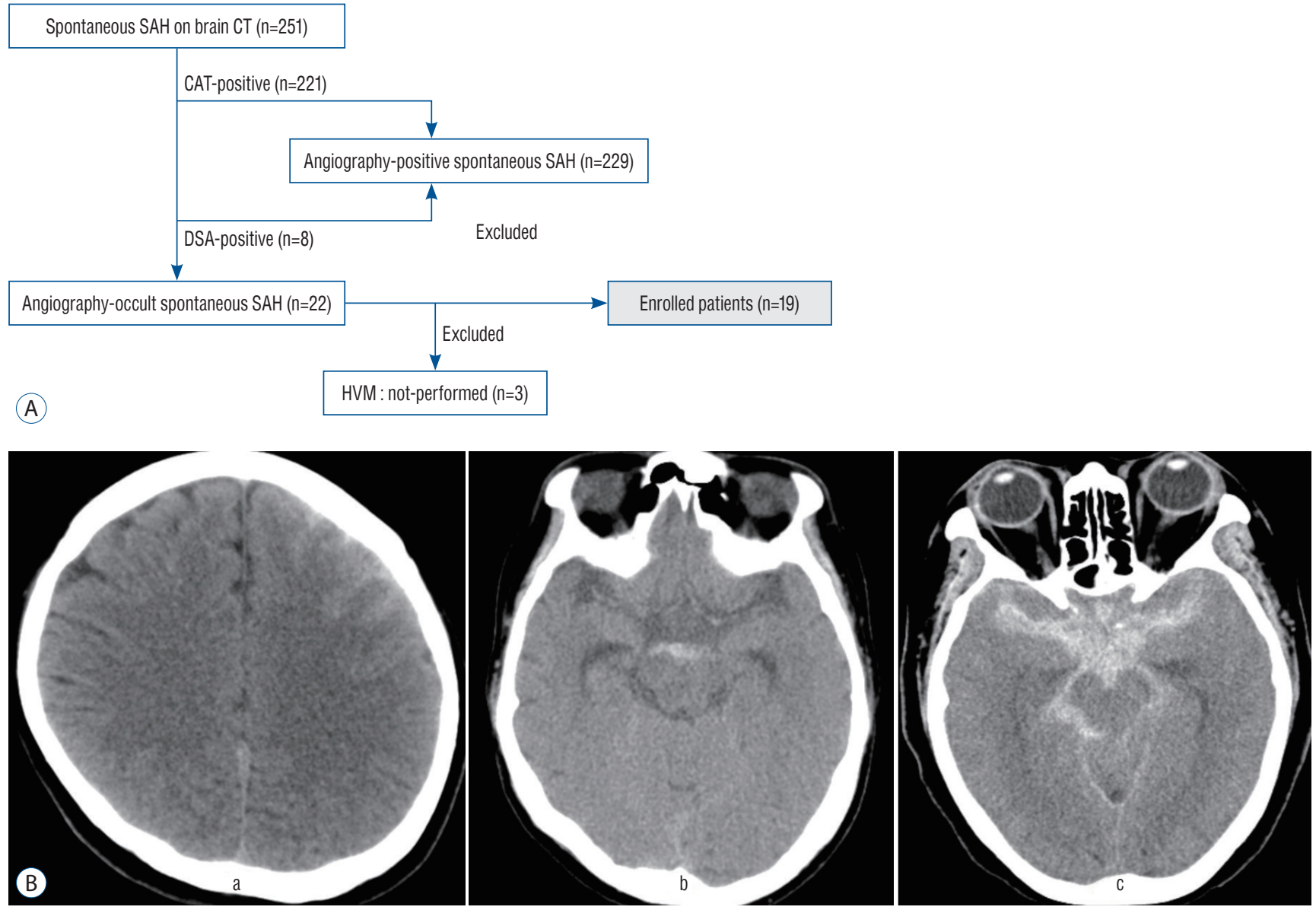

Fig. 1. A : A flowchart representing the process of patient recruitment. B : The representative case illustration of three types of subarachnoid hemorrhage (SAH). A typical case of convexal type of SAH shows hemorrhage isolated in one or several adjacent sulci of left cerebral hemisphere without blood at the basal cisterns, interhemispheric fissure ventricle or parenchyma (a). Perimensencephalic type of SAH case demonstrates hemorrhage loculated in cisternal space of midbrain and the absence of a clot in other space (b). Diffuse type SAH indicates hemorrhage distributed widely in basal, interhemispheric, sylvian and perimesencephalic cisterns, which resembles a diffuse SAH caused by aneurysmal rupture (c). CT : computed tomography, CTA : computed tomography angiography, DSA : digital subtraction angiography, HVM : high-resolution vessel wall magnetic resonance imaging. 
suggest that there might be a hidden arteriopathy, which has not been unveiled.

Recently, three-dimensional (3D) high-resolution vessel wall MRI (HVM) is used to validate arterial wall pathologies such as dissection, aneurysmal wall stability, and the nature of plaque. The information of (pathologic) morphology of the arterial wall can be demonstrated on the 3D-HVM but not on the conventional angiography which only reflect the intraluminal blood flow ${ }^{2,26)}$. This study aimed to reconsider the role of the HVM in investigating the arterial pathology of AOsSAH by illuminating the walls of the cerebral arteries.

\section{MATERIALS AND METHODS}

Ethical approval for this study was obtained from the Institutional Ethics Review Board of Guro Hospital, Korea University (approval number : 2020GR0299). The requirement for informed consent was waived because of the retrospective nature of the study. Data were extracted retrospectively from our prospective database, which includes surgical records, videos, and source angiography data of patients with SAH.

\section{Patient enrolment}

We retrospectively reviewed 251 patients who admitted to our hospital between January 2017 and December 2020 and were diagnosed with spontaneous SAH. Of them, 229 patients were diagnosed with definitive arteriopathy (aneurysm or other causes) on the initial angiographic evaluation. Twentytwo patients with negative findings on the initial angiographic evaluations, including computed tomography (CT) angiography and complete six-vessel cerebral angiography, were considered to have AOsSAH. Among them, three patients were excluded because they did not undergo 3D-HVM due to their poor conditions. Finally, 19 patients, who were diagnosed with AOsSAH in initial evaluations and underwent 3D-HVM during acute or the subacute phase, were enrolled (Fig. 1A).

\section{Classification of SAH}

Enrolled patients were classified as three groups according to the distribution and amount of their hemorrhages : convexal $(n=2)$ or perimesencephalic $(n=4)$, and diffuse type $(n=13)$. Convexal SAH was defined as isolated hemorrhage in one or several adjacent sulci in the absence of blood at the bas- al cisterns, interhemispheric fissure, ventricle, or parenchy$\mathrm{ma}^{12)}$. According to the definition proposed by Rinkel et al. ${ }^{23)}$, perimesencephalic SAH was defined as a hemorrhage located immediately anterior to the midbrain with or without extension of blood into the ambient cistern or the Sylvian fissure

Table 1. Imaging parameters for high-resolution vessel wall imaging of the large intracranial arteries

\begin{tabular}{|c|c|c|c|}
\hline & TOF & T2 SPACE & T1 SPACE \\
\hline Scan time & $\begin{array}{l}6 \text { minutes } \\
58 \text { seconds }\end{array}$ & $\begin{array}{l}7 \text { minutes } \\
27 \text { seconds }\end{array}$ & $\begin{array}{l}7 \text { minutes } \\
22 \text { seconds }\end{array}$ \\
\hline Slabs & 10 & 1 & 1 \\
\hline TR (ms) & 23 & 1200 & 600 \\
\hline $\mathrm{TE}$ (ms) & 3.98 & 124 & 12 \\
\hline FOV, read×phase (mm) & $210 \times 158$ & $160 \times 160$ & $160 \times 160$ \\
\hline Matrix, freq. $\times$ phase & $448 \times 314$ & $320 \times 288$ & $320 \times 288$ \\
\hline Slice thickness (mm) & 0.45 & 0.5 & 0.5 \\
\hline Slice resolution (\%) & 50.0 & 100.0 & 100.0 \\
\hline
\end{tabular}

TOF : time of flight, SPACE : sampling perfection with applicationoptimized contrasts by using different angle evolutions, TR : repetition time, TE : echo time, FOV : field of view, freq. : frequency

Table 2. Demographics of enrolled patients

\begin{tabular}{lc}
\hline & Value $(\mathbf{n}=\mathbf{1 9})$ \\
\hline Sex, female & $13(68.4)$ \\
Age (years) & $55.36 \pm 14.24$ \\
Type of AOsSAH & \\
Perimesencephalic & $4(21.1)$ \\
Convexal & $2(10.5)$ \\
Diffuse & $13(68.4)$ \\
Modified Fisher grade & \\
॥ & $4(21.1)$ \\
III & $11(57.8)$ \\
IV & $4(21.1)$ \\
Ventriculomegaly or hydrocephalus & $5(26.3)$ \\
HVM evaluation, days after onset & $4.74 \pm 5.21$ \\
mRS, final & $17(89.4)$ \\
0, no symptom & $1(5.3)$ \\
1, mild symptom without disability & $1(5.3)$ \\
4, severe disability &
\end{tabular}

Values are presented as mean \pm standard deviation or number (\%). AOsSAH : angiography occult spontaneous subarachnoid hemorrhage, HVM : high-resolution vessel wall magnetic resonance imaging, mRS: modified Rankin Scale 
and the absence of a clot in the anterior interhemispheric fissure or the intraventricular space. Hemorrhages that did not fulfil the aforementioned criteria and distributed diffusely in basal and Sylvian cisterns were classified as diffuse $\mathrm{SAH}^{3)}$. The illustrative images of three types of AOsSAH are presented in Fig. 1B.

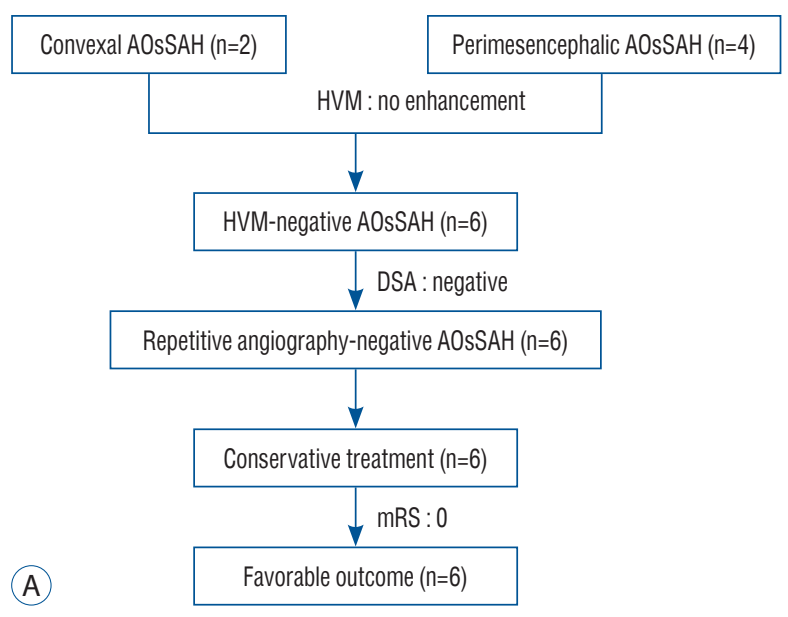

\section{Clinical and radiologic data}

Medical records of the enrolled patients were retrospectively reviewed including age, sex, hidden trauma histories, laboratory findings, clinical courses, and final modified Rankin Scale (mRS) scores. Every radiologic image was again reviewed. All patients underwent laboratory workups to reveal hidden malignancies, coagulopathies, and autoimmune diseases such as vasculitis. Brain and complete spinal MRIs were

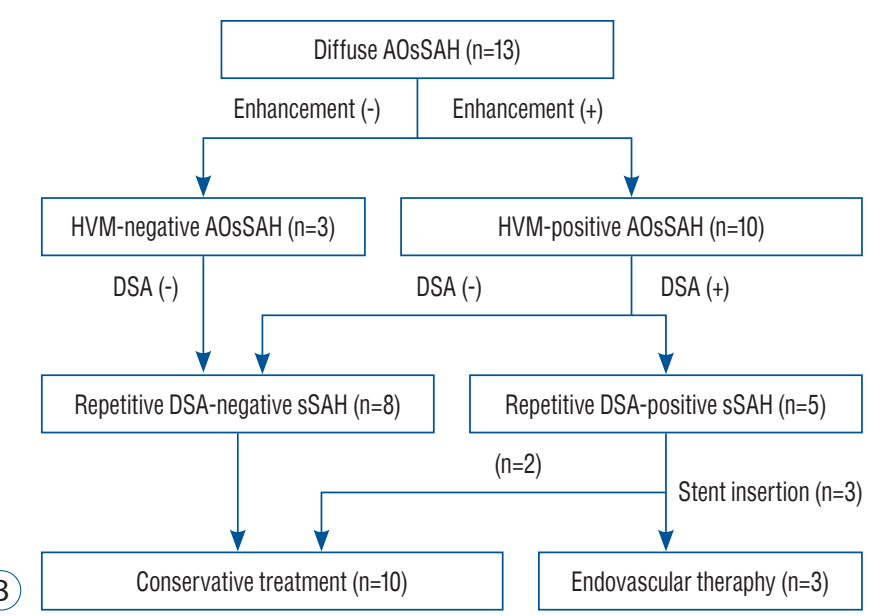

Fig. 2. A flowchart of the enrolled patients. A : A flowchart showing evaluation and treatment process of convexal and perimesencephalic type subarachnoid hemorrhage patients. B : A flowchart showing evaluation and treatment process of diffuse type subarachnoid hemorrhage patients. AOsSAH : angiographically occult spontaneous subarachnoid hemorrhage, HVM : high-resolution vessel wall magnetic resonance imaging, DSA : digital subtraction angiography, sSAH: spontaneous subarachnoid hemorrhage.

Table 3. Characteristics of patients with HVM-positive AOsSAH

\begin{tabular}{|c|c|c|c|c|c|c|c|c|c|}
\hline Age (years) & Sex & $\begin{array}{c}\text { Type of } \\
\text { SAH }\end{array}$ & $\begin{array}{l}\text { Fisher } \\
\text { grade }\end{array}$ & $\begin{array}{l}\text { Date of HVM } \\
\text { performance }\end{array}$ & $\begin{array}{c}\text { Location of } \\
\text { enhancement on HVM } \\
\text { (days from SAH) }\end{array}$ & $\begin{array}{l}\text { Findings on } \\
\text { subsequent } \\
\text { angiography }\end{array}$ & Final diagnosis & Treatment & $\mathrm{mRS}$ \\
\hline 73 & M & $\mathrm{D}$ & 3 & 2 & $\mathrm{BA}(2)$ & $\begin{array}{l}\text { New stenosis on BA } \\
\text { trunk }\end{array}$ & $\begin{array}{l}\text { R/O dissection, } \\
\text { BA }\end{array}$ & Observation & 0 \\
\hline 65 & M & D & 3 & 1 & $\mathrm{BA}(1)$ & Negative & AOsSAH & Observation & 0 \\
\hline 57 & M & D & 4 & 3 & MCA Rt (3) & New stenosis, M1 Rt & Dissection, M1 Rt & Observation & 0 \\
\hline 62 & M & D & 4 & 5 & ICA Rt (5) & BBA, ICA Rt & BBA, ICA Rt & Multiple stents & 0 \\
\hline 57 & M & $\mathrm{D}$ & 3 & 10 & BA (10) & $\begin{array}{l}\text { Perforator aneurysm, } \\
\text { BA }\end{array}$ & $\begin{array}{l}\text { Perforator } \\
\text { aneurysm, BA }\end{array}$ & Multiple stents & 0 \\
\hline 44 & $\mathrm{~F}$ & $D$ & 3 & 21 & $\mathrm{BA}(21)$ & Negative & AOsSAH & Observation & 0 \\
\hline 48 & F & $\mathrm{D}$ & 3 & 1 & MCA Rt (1) & Negative & AOsSAH & Observation & 0 \\
\hline 63 & $\mathrm{~F}$ & $D$ & 3 & 4 & $\mathrm{BA}(4)$ & Negative & AOsSAH & Observation & 1 \\
\hline 61 & $\mathrm{~F}$ & $D$ & 3 & 0 & ICA Rt (0) & BBA, ICA Rt & BBA, ICA Rt & Single stent & 0 \\
\hline 58 & $\mathrm{~F}$ & D & 3 & 3 & $\mathrm{BA}(3)$ & Negative & AOsSAH & Observation & 0 \\
\hline
\end{tabular}

HVM : high-resolution vessel wall magnetic resonance imaging, AOsSAH : angiographically occult spontaneous subarachnoid hemorrhage, SAH : subarachnoid hemorrhage, $m$ RS : modified Rankin Scale, $M$ : male, D : diffuse type of SAH, BA : basilar artery, R/O : rule-out, MCA : middle cerebral artery, Rt : right, $\mathrm{M} 1$ : first segment of the middle cerebral artery, ICA : internal cerebral artery, BBA : blood blister-like aneurysm, $\mathrm{F}:$ female 
Table 4. Number of catheter angiography and time of notification of angiographic lesion

\begin{tabular}{|c|c|}
\hline $\begin{array}{l}\text { No. of catheter } \\
\text { angiography (CTA, MRA) }\end{array}$ & $\begin{array}{c}\text { Date of notification of } \\
\text { angiographic lesion, modality }\end{array}$ \\
\hline $2(2,1)$ & 14 , second CTA \\
\hline \multicolumn{2}{|l|}{$2(2,1)$} \\
\hline $3(1,1)$ & 4, MRA \\
\hline $3(1,1)$ & 14 , third angiography \\
\hline $3(1,1)$ & $\begin{array}{l}\text { 5, second angiography (retrospective } \\
\text { re-analysis of 3-dimension angiography } \\
\text { according to HVM) }\end{array}$ \\
\hline \multicolumn{2}{|l|}{$2(1,1)$} \\
\hline \multicolumn{2}{|l|}{$1(2,1)$} \\
\hline \multicolumn{2}{|l|}{$4(2,1)$} \\
\hline $3(1,1)$ & 5, second angiography \\
\hline $3(1,1)$ & \\
\hline
\end{tabular}

No. of catheter angiography (CTA, MRA) : number of performance of catheter angiography (number of performance of CTA, number of performance of MRA). CTA : computed tomography angiography, MRA magnetic resonance angiography, HVM : high-resolution vessel wall magnetic resonance imaging performed to determine other possible causes of SAH. Patients underwent contrast-enhanced 3D-HVM. Two certified neuroradiologists blinded to the clinical data independently evaluated the 3D-HVM findings. Abnormal arterial wall enhancement was defined as the presence of a moderate-tostrong arterial wall enhancement in the relevant location of the hematoma observed by comparing the pre-gadolinium and post-gadolinium T1-weighted images ${ }^{20)}$. Disagreements between the two radiologists were resolved by consensus. All patients underwent one or more subsequent angiographic evaluation(s) regardless of the HVM findings. In patients with positive findings on HVM, special attention was paid to the enhancing lesion with high-resolution 3D reconstruction of rotational angiography images (Fig. 1B). The radiological and clinical data were analyzed retrospectively.
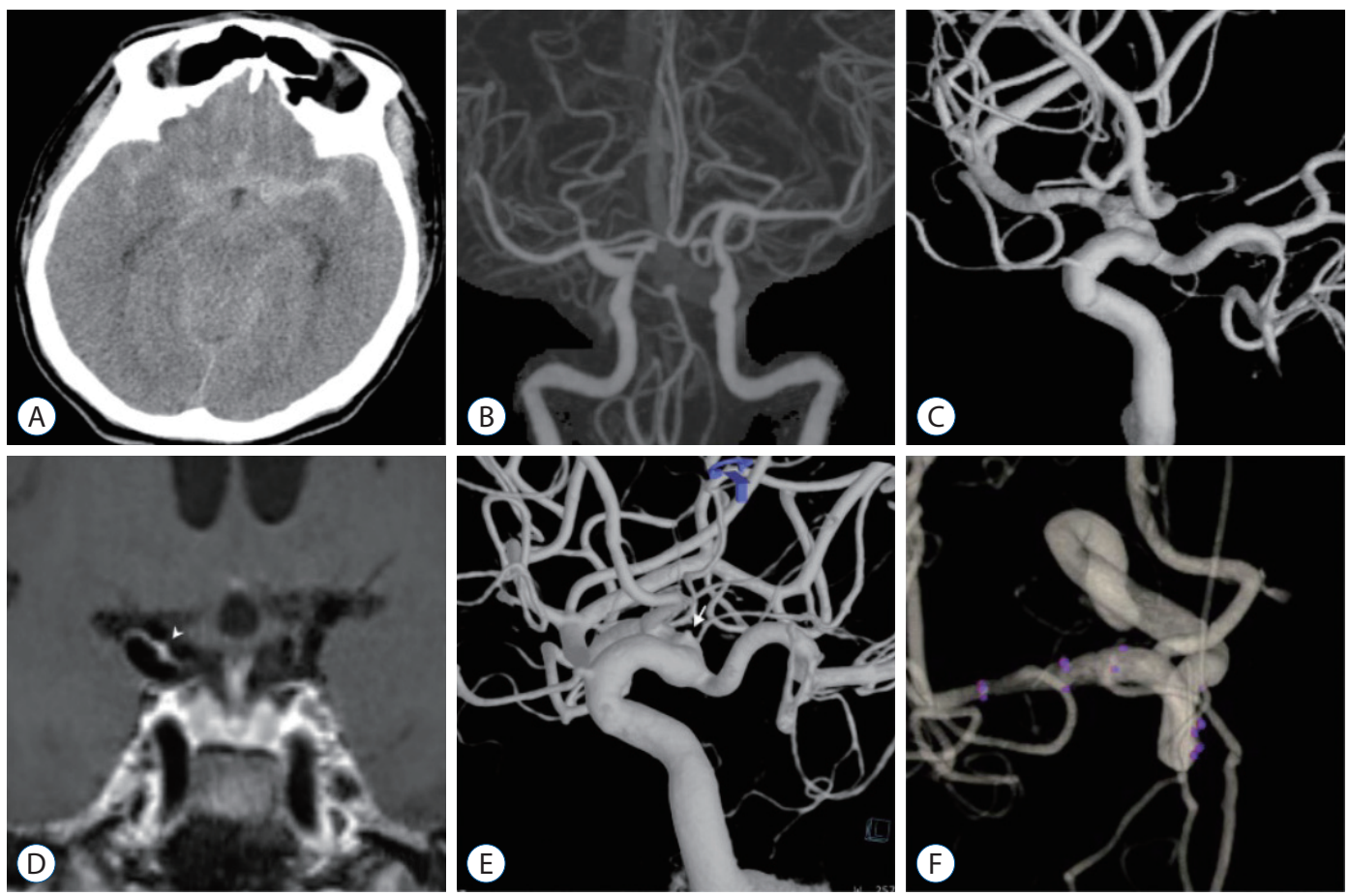

Fig. 3. A case of ruptured blood blister-like aneurysm on right internal cerebral artery. A : A 62-year-old man was admitted in the emergency room complaining thunderclap worst headache. Brain computed tomography (CT) scan image shows diffuse type subarachnoid hemorrhage. The hemorrhage amount is slightly larger in left sylvian fissure than right side. B : The initial CT angiography shows no definitive arteriopathy causing subarachnoid hemorrhage. C : Six-vessel complete angiography taken on the first day of admission failed to discover any definitive vascular cause. D : Three-dimensional high-resolution vessel wall magnetic resonance imaging (MRI) taken on the fifth day of admission demonstrates strong focal enhancement on the dorsomedial wall of right side distal internal cerebral artery (white arrowhead). This finding suggests possibility of ruptured arteriopathy at this region. $\mathrm{E}$ : The second week angiography also failed to show any vasculopathy. The third week angiography high resolution 3-dimensional reconstruction guided by the finding from vessel wall MRI shows a blood blister-like aneurysm on the same location of strong enhancement (white arrow). This lesion was treated with overlapped laser cut stents. F : Six months follow-up angiography reveals complete healing of the ruptured blood blister-like aneurysm. 

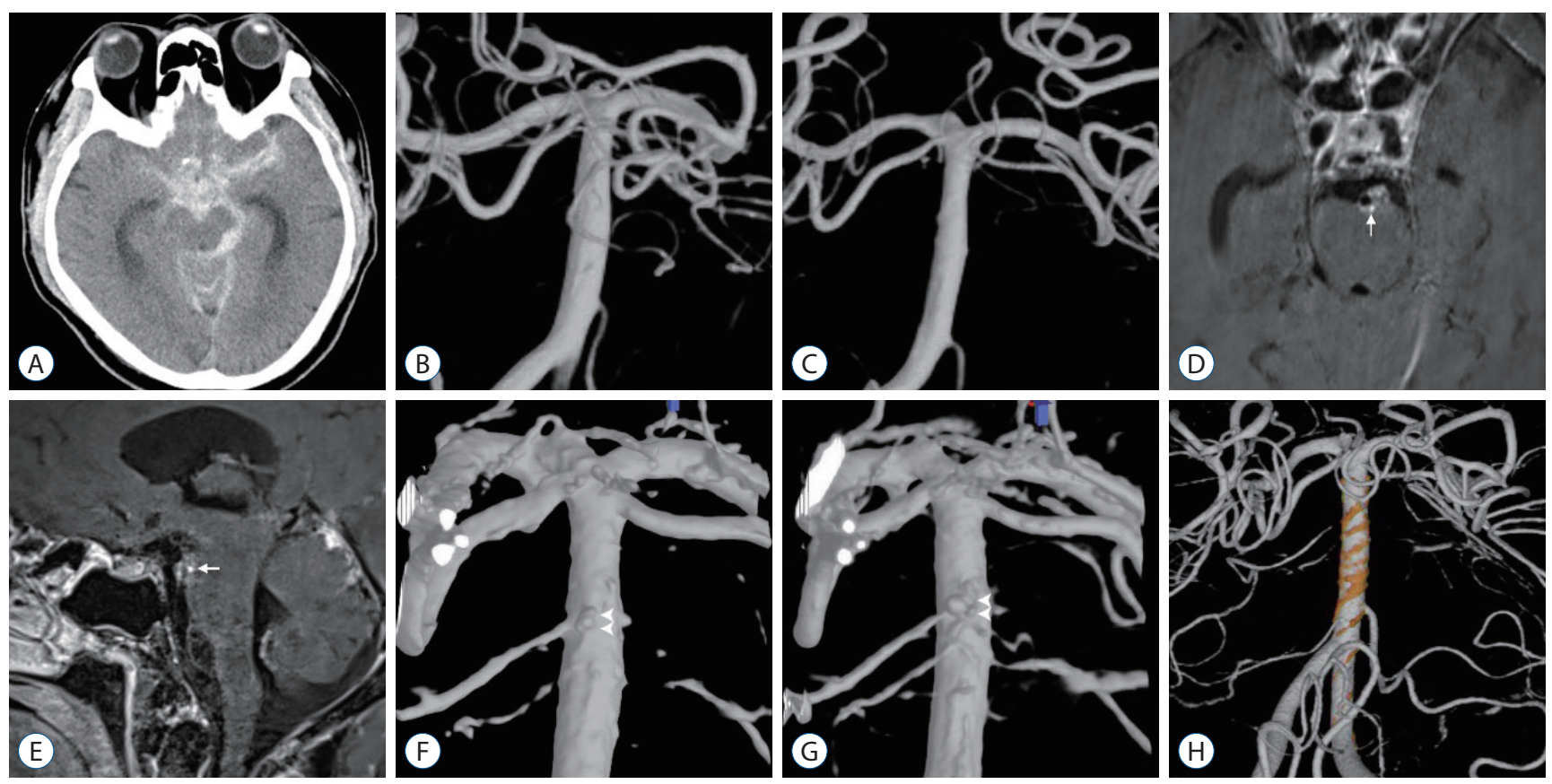

Fig. 4. A case of ruptured perforator or blood blister-like aneurysm on posterior wall of distal basilar artery. A-C : Initial evaluation images showing diffuse subarachnoid hemorrhage on computed tomography scan and no definitive arteriopathy on first and second angiography. D and E : Threedimensional high-resolution vessel wall magnetic resonance imaging (MRI) taken on the 10th day of admission demonstrates strong nodular enhancement on the posterior wall of basilar artery (white arrow). F : Re-do high-resolution 3-dimensional reconstruction of second angiography according to the vessel wall MRI finding reveals a tiny aneurysm on the posterior wall of the basilar artery at the concordant location of nodular enhancement on vessel wall MRI (white arrowheads). G : Third angiography shows definitive aneurysm on the same location (white arrowheads). $H$ : This image shows the result of endovascular treatment using overlapped braided stents.

\section{Protocol of 3D-HVM}

The imaging protocol of 3D-HVM included the following: 1) a 3 T scanner (Skyra; Siemens, Erlangen, Germany); 2) time of flight magnetic resonance angiography for the circle of Willis; 3) T2-weighted imaging and pre-gadolinium and postgadolinium enhanced T1-weighted sagittal imaging using isotropic 3D sampling perfection with application optimized contrast using different flip angle evolutions sequences with blood suppression for entire intracranial large arteries; and 4) multiplanar reconstruction for coronal or axial acquisition. The detailed imaging parameters are described in Table 1.

\section{Statistical analysis}

Continuous variables are expressed as mean \pm standard deviation, while categorical variables are expressed as frequency (percentage). Statistical comparison analysis was not performed because of the small sample size.

\section{RESULTS}

\section{General demographics}

Among the patients with sSAH ( $\mathrm{n}=251), 22$ patients were confirmed as AOsSAH in the initial angiographic evaluations of computed tomography angiography (CTA) and digital subtraction angiography (DSA) (8.76\%). Among 19 enrolled patients, patients were classified as having convexal $(n=2)$, perimesencephalic $(n=4)$, and diffuse $(n=13)$ AOsSAHs. Baseline characteristics of the enrolled patients were shown in Table 2. Female predominance (68.4\%), mean age of 55-year-old, $26.5 \%$ of ventriculomegaly or hydrocephalus, and $5.3 \%$ of poor outcome ( $\mathrm{n}=1$; mRS score, 4$)$ were noted.

Eighteen patients underwent 3D-HVM evaluation in the acute phase within 10 days of SAH onset during hospitalization, while one patient underwent 3D-HVM evaluation in the subacute phase (days 21 of SAH onset).

\section{Convexal or perimesencephalic AOsSAH}

Six patients were diagnosed with convexal or perimesence- 
phalic AOsSAH (31.6\%). Only the initial brain CT revealed the specific types of AOsSAH and every other radiologic image were noted as negative (CTA, initial DSA, 3D-HVM, spine and brain MRI, and repetitive DSAs). All patients with perimesencephalic and convexal AOsSAH showed excellent outcomes (mRS score, 0 ) with an uneventful course. The clinical courses of the patients were shown in Fig. 2A.

\section{Diffuse AOsSAH}

Thirteen patients were classified as diffuse-type AOsSAH (68.4\%). They all underwent 3D-HVM and 10 patients showed positive findings of abnormal focal enhancement on the cerebral vessel wall (10/13, 76.9\%). All patients underwent one or more additional follow-up angiographic evaluation(s) with high-resolution 3D reconstruction, the results of which were intensively interpreted with special attention to the location of the enhancement. Among 10 patients with HVM-positive enhancement, five patients showed angiographic abnormalities at the concordant locations of enhancement and hematoma sites on the initial CT (50\%). The flowchart of the evaluations was shown in Fig. 2B. In terms of outcomes, two patients with diffuse AOsSAH had an eventful course with symptomatic vasospasm and hydrocephalus (mRS score, 1 and 4; 15.4\%).

Tables 3 and 4 present the detailed information of HVMpositive patients $(n=10)$. They all were in the diffuse-type AOsSAH and their initial Fisher's grade was 3 or 4 . Focal enhancement was noted in basilar artery $(\mathrm{BA} ; \mathrm{n}=6)$, internal carotid artery $(\mathrm{n}=2)$, or middle cerebral artery $(\mathrm{n}=2)$. Among 10 HVM-positive lesions, five were confirmed as pathologic in repetitive DSA (50\%) : one definitive and one suspicious dissection $(n=2)$, blood blister-like aneurysm $(n=2)$, and small perforating artery aneurysm $(n=1)$. The latter three patients eventually underwent endovascular treatment. And a dissection on middle cerebral artery trunk case was conservatively treated according to its benign clinical course and angiographic finding of no aneurysmal dilatation without progression to severe stenosis causing ischemia.

\section{Illustrative cases}

Two patients with strong enhancement of the medial wall of the supraclinoid internal carotid artery (non-branching site) were diagnosed with a blood blister-like aneurysm at the corresponding location. Their initial angiography did not reveal any vasculopathy and 3D-HVM findings lead to intense in- vestigation on the second week follow-up angiography with $3 \mathrm{D}$ reconstruction and final diagnosis was made as ruptured blood blister-like aneurysm. These lesions were treated with overlapped Enterprise stents (Cordis Neurovascular, Miami, FL, USA) (Fig. 3).

Focal enhancement of the BA trunk was observed in six patients. Of them, one patient was found to have a blood blisterlike or perforator aneurysm at the relevant spot of 3D-HVM enhancement on subsequent angiography. Weekly angiographic follow-up evaluations demonstrated increase in the size of the aneurysm, which was eventually treated with overlapped low-profile visualized intraluminal support (MicroVention-Terumo, Tustin, CA, USA) stent placement. Flow diverter stent was not considered according to the guidelines of Korean Insurance System (Fig. 4).

\section{DISCUSSION}

In this study, AOsSAH showed a high rate of focal abnormal arterial wall enhancement at the corresponding hematoma site on the 3D-HVM workup protocol in 10 of 19 patients (52.6\%). In particular, in diffuse AOsSAH, strong enhancement of the relevant area of the cerebral artery was observed with a high incidence (76.9\%). Subsequent angiographic evaluations revealed arteriopathies at the enhancement region on $3 \mathrm{D}-\mathrm{HVM}$ in five patients $(50 \%$ patients with positive $3 \mathrm{D}$ HVM findings). Further, 3D-HVM was helpful in the detection of arteriopathies that were not visible on thorough angiographic evaluation in the early phase of the disease. 3D-HVM guided subsequent focused and intense evaluation for determining the causative arteriopathy.

The incidence of AOsSAH is approximately $20 \%$ in clinical practice ${ }^{14)}$. Repeated angiography and MRIs of brain and spine are used to assess the vascular causes of AOsSAH. However, the yield rate of serial angiography is low (approximately 7-15\%), and the detection rate of central nervous system MRI is even lower (approximately $4-8 \%)^{9-11,27,28)}$.

The etiology of perimesencephalic SAH has not been determined. A non-arterial source of hemorrhage has been proposed because of the sparse amount of hemorrhage and a relatively benign clinical course compared to aneurysmal SAH. Variants of the venous system such as the basal vein of Rosenthal have been suggested to be associated with this type of 
$\mathrm{SAH}^{4,10,25)}$.

Although the clinical outcome is generally favorable, 20\% patients with AOsSAH exhibit poor clinical outcomes or events such as delayed cerebral ischemia, hydrocephalus, rebleeding, and neurological sequelae $e^{1,5,12)}$. Large quantity of hemorrhage (Fisher grade $>3$ ) is associated with poor prognosis $^{13)}$. In particular, diffuse AOsSAH exhibits poor clinical outcomes $^{15)}$. Diffuse AOsSAH exhibits a clinical situation similar to aneurysmal SAH in terms of significant amount of hematoma with acute clinical demonstration, suggesting an arterial etiology for this type of AOsSAH. This prompts clinicians to perform repetitive angiographic evaluations to identify any evidence of arteriopathy. Angiography may yield falsenegative results because of vascular thrombosis, mass effect by an adjacent hematoma, vasospasm, or technical inadequacy ${ }^{12}$. Therefore, repeated angiographic evaluation is considered mandatory. However, the detection rate of angiography for the contributing arteriopathy is low (approximately 15\%) ${ }^{11,12,28)}$.

Even with unprecedented technological developments in angiography, it has critical limitations. Angiography cannot provide information beyond the arterial lumen. Recently, HVM techniques have been developed to subtract the blood flow signal from the vascular lumen. This process enables direct visualization of the vessel wall with enhanced differentiation among diverse cerebrovasculopathies ${ }^{26)}$. Although interpretation of an HVM image is challenging and occasionally controversial, many reports have advocated its practicality. Recent studies have indicated that vulnerability of intracranial atheromatous plaque, presence of dissection, instability of unruptured aneurysms, and detection of ruptured aneurysms in cases of simultaneous multiple aneurysms can be validated using $\mathrm{HVM}^{2,7,16,19,20,26)}$. Chung et al. ${ }^{6)}$ have published an interesting case series involving in vivo HVM investigation of hidden pathologic causes of various vertebral arterial diseases. Thus, vessel wall imaging has been extensively used as a complementary tool to luminal imaging modalities in cerebrovascular diseases. Our study also demonstrated the usefulness of vessel wall imaging in determining the possible cause of AOsSAH. In our study, 3D-HVM revealed a high proportion (76.9\%) of lesions with abnormal enhancement in diffuse AOsSAH. Despite the small sample size, the results of consecutive patient data are encouraging and interesting. HVM clearly escalated the detection rate of vasculopathy in the subsequent evaluation by providing an opportunity to pay close attention to the specific region of the vessel wall. Ten patients were suspected to have a causative arteriopathy on 3D-HVM evaluation. Of them, contributing arteriopathy was confirmed in five patients $(26.3 \%$ patients with all types of AOsSAH, $38.5 \%$ patients with diffuse $\mathrm{AOsSAH}$, and 50\% patients with positive findings on 3D-HVM) in subsequent angiography and three patients underwent definitive endovascular treatment. HVM did not change the treatment strategy in all cases, but it considerably contributed to the detection of vasculopathy in limited cases. Considering the rebleeding risk of up to $8.5 \%$ and a relatively large amount of hemorrhage in diffuse $\mathrm{AOsSAH}^{15)}$, the importance of information about the status of the arterial wall should not be overlooked. 3D-HVM provides physicians with a new insight - the arterial wall status - for the investigation of the usual suspects in AOsSAH.

Controversies regarding the interpretation of wall enhancement are anticipated. However, comparison of the images obtained in the T1, T2, and T1 enhancement protocols in our study enabled us to distinguish pathologic focal enhancement from enhancement that could be observed in adjacent hematoma or in atherosclerosis of the wall. Although we did not present the related data, three patients with diffuse AOsSAH and positive HVM findings who underwent conservative treatment were followed up with the same 3D-HVM protocol at 2 or 4 months after the onset of SAH; all demonstrated disappearance or waning of the previous enhanced lesions. This phenomenon in the chronic phase may imply that focal enhancement of relevant corresponding vessel wall in the acute or the subacute phase of AOsSAH could be considered a pathological change, which was either the cause or the result of SAH. The disappearance of the lesions also suggests that if they were the cause of the hemorrhage, they could heal spontaneously.

Recent studies have suggested that circumferential or strong focal enhancement of the aneurysm wall indicates an increased risk of unstable aneurysm ${ }^{7,16)}$. Moreover, in the setting of SAH with multiple aneurysms, the culprit aneurysm has a tendency to exhibit enhancement circumferentially or focally at the rupture point ${ }^{7,20)}$. Natori et al. ${ }^{21)}$ have demonstrated the usefulness of 3D-HVM in detection of intramural high-intensity signal in acute vertebrobasilar dissection in a prospectively designed study that compared 3D-HVM with conventional magnetic resonance angiography. These observations support the potential of our results in suggesting that focal wall en- 
hancement indicates a high possibility of arterial cause in AOsSAH, especially in the diffuse type.

Park et al. ${ }^{22}$ have demonstrated that the final angiographic diagnosis requires 3D angiographic evaluation. In our study, routine two-dimensional angiography could not demonstrate any abnormalities in the vessel wall, but the highly reconstructed 3D images revealed definitive arteriopathies in five patients with angiographic confirmation. Further, 3D-HVM played an important guiding role in thorough evaluation of the concordant lesions with repeated 3D angiography evaluation performed every week.

The study limitations include the small number of patients and the retrospective nature of the study. However, considering the consecutive nature of the patient data in our hospital, we believe that 3D-HVM in the acute phase of AOsSAH provided a valuable insight into the cause of hemorrhage.

\section{CONCLUSION}

3D-HVM in the acute phase of AOsSAH showed a high detection rate for abnormal focal wall enhancement in the cerebral arteries concordant with the responsible hematoma region, especially in cases of diffuse AOsSAH. Moreover, it provided resourceful guidance for further evaluation and subsequent treatment. The study findings may provide valuable evidence and insights for the research regarding the pathogenesis of AOsSAH.

\section{CONFLICTS OF INTEREST}

No potential conflict of interest relevant to this article was reported.

\section{INFORMED CONSENT}

This type of study does not require informed consent.

\section{AUTHOR CONTRIBUTIONS}

\author{
Conceptualization: WY, THK
}

\author{
Data curation : WY, HR \\ Formal analysis: WY \\ Methodology: WY, JHK \\ Project administration : WY \\ Visualization: WY \\ Writing - original draft: WY \\ Writing - review \& editing: WY, THK
}

\section{ORCID}
Wonki Yoon
https://orcid.org/0000-0003-3715-0718
Jang Hun Kim
https://orcid.org/0000-0001-8557-1627
Haewon Roh
https://orcid.org/0000-0002-7527-4407
Taek-Hyun Kwon https://orcid.org/0000-0002-8428-6952

\section{References}

1. Beseoglu K, Pannes S, Steiger HJ, Hänggi D : Long-term outcome and quality of life after nonaneurysmal subarachnoid hemorrhage. Acta Neurochir (Wien) $152:$ 409-416, 2010

2. Bhogal $P$, Uff $C$, Makalanda $H L$ : Vessel wall MRI and intracranial aneurysms. J Neurointerv Surg 8 : 1160-1162, 2016

3. Boswell S, Thorell W, Gogela S, Lyden E, Surdell D : Angiogram-negative subarachnoid hemorrhage: outcomes data and review of the literature. J Stroke Cerebrovasc Dis 22 : 750-757, 2013

4. Buyukkaya R, Yıldırım N, Cebeci H, Kocaeli H, Dusak A, Ocakoğlu G, et al. : The relationship between perimesencephalic subarachnoid hemorrhage and deep venous system drainage pattern and calibrations. Clin Imaging 38 : 226-230, 2014

5. Cánovas D, Gil A, Jato M, de Miquel M, Rubio F : Clinical outcome of spontaneous non-aneurysmal subarachnoid hemorrhage in 108 patients. Eur J Neurol $19:$ 457-461, 2012

6. Chung JW, Kim BJ, Choi BS, Sohn CH, Bae HJ, Yoon BW, et al. : Highresolution magnetic resonance imaging reveals hidden etiologies of symptomatic vertebral arterial lesions. J Stroke Cerebrovasc Dis 23 : 293-302, 2014

7. Edjlali M, Gentric JC, Régent-Rodriguez C, Trystram D, Hassen WB, Lion $S$, et al. : Does aneurysmal wall enhancement on vessel wall MRI help to distinguish stable from unstable intracranial aneurysms? Stroke 45 : 3704-3706, 2014

8. Germans MR, Coert BA, Majoie CB, van den Berg R, Lycklama À Nijeholt $G$, Rinkel $G$, et al. : Yield of spinal imaging in nonaneurysmal, nonperimesencephalic subarachnoid hemorrhage. Neurology 84 : 13371340, 2015

9. Kalra VB, Wu X, Matouk CC, Malhotra A : Use of follow-up imaging in isolated perimesencephalic subarachnoid hemorrhage: a meta-analysis. 
Stroke 46 : 401-406, 2015

10. Kapadia A, Schweizer TA, Spears J, Cusimano M, Macdonald RL : Nonaneurysmal perimesencephalic subarachnoid hemorrhage: diagnosis, pathophysiology, clinical characteristics, and long-term outcome. World Neurosurg 82 : 1131-1143, 2014

11. Khan AA, Smith JD, Kirkman MA, Robertson FJ, Wong K, Dott C, et al. : Angiogram negative subarachnoid haemorrhage: outcomes and the role of repeat angiography. Clin Neurol Neurosurg 115 : 1470-1475, 2013

12. Kim YW, Lawson MF, Hoh BL : Nonaneurysmal subarachnoid hemorrhage: an update. Curr Atheroscler Rep 14 : 328-334, 2012

13. Konczalla J, Kashefiolasl S, Brawanski N, Lescher S, Senft C, Platz J, et al. : Cerebral vasospasm and delayed cerebral infarctions in 225 patients with non-aneurysmal subarachnoid hemorrhage: the underestimated risk of Fisher 3 blood distribution. J Neurointerv Surg 8 : 1247-1252, 2016

14. Konczalla J, Kashefiolasl S, Brawanski N, Senft C, Seifert V, Platz J : Increasing numbers of nonaneurysmal subarachnoid hemorrhage in the last 15 years: antithrombotic medication as reason and prognostic factor? J Neurosurg 124 : 1731-1737, 2016

15. Konczalla J, Schuss P, Platz J, Vatter H, Seifert V, Güresir E : Clinical outcome and prognostic factors of patients with angiogram-negative and non-perimesencephalic subarachnoid hemorrhage: benign prognosis like perimesencephalic SAH or same risk as aneurysmal SAH? Neurosurg Rev 38 : 121-127; discussion 127, 2015

16. Lehman VT, Brinjikji W, Kallmes DF, Huston J 3rd, Lanzino G, Rabinstein $A A$, et al. : Clinical interpretation of high-resolution vessel wall MRI of intracranial arterial diseases. Br J Radiol 89 : 20160496, 2016

17. Macdonald RL, Schweizer TA : Spontaneous subarachnoid haemorrhage. Lancet 389 : 655-666, 2017

18. Marder CP, Narla V, Fink JR, Tozer Fink KR : Subarachnoid hemorrhage: beyond aneurysms. AJR Am J Roentgenol 202 : 25-37, 2014

19. Mossa-Basha M, Alexander M, Gaddikeri S, Yuan C, Gandhi D : Vessel wall imaging for intracranial vascular disease evaluation. J Neuroin- terv Surg 8 : 1154-1159, 2016

20. Nagahata $S$, Nagahata $M$, Obara $M$, Kondo $R$, Minagawa $N$, Sato $S$, et al. : Wall enhancement of the intracranial aneurysms revealed by magnetic resonance vessel wall imaging using three-dimensional turbo spin-echo sequence with motion-sensitized driven-equilibrium: a sign of ruptured aneurysm? Clin Neuroradiol 26 : 277-283, 2016

21. Natori T, Sasaki M, Miyoshi M, Ohba H, Oura MY, Narumi S, et al. : Detection of vessel wall lesions in spontaneous symptomatic vertebrobasilar artery dissection using T1-weighted 3-dimensional Imaging. J Stroke Cerebrovasc Dis 23 : 2419-2424, 2014

22. Park SQ, Kwon OK, Kim SH, Oh CW, Han MH : Pre-mesencephalic subarachnoid hemorrhage: rupture of tiny aneurysms of the basilar artery perforator. Acta Neurochir (Wien) 151 : 1639-1646, 2009

23. Rinkel GJ, Wijdicks EF, Vermeulen M, Ramos LM, Tanghe HL, Hasan D, et al. : Nonaneurysmal perimesencephalic subarachnoid hemorrhage: CT and MR patterns that differ from aneurysmal rupture. AJNR Am J Neuroradiol $12: 829-834,1991$

24. Rogg JM, Smeaton S, Doberstein C, Goldstein JH, Tung GA, Haas RA : Assessment of the value of MR imaging for examining patients with angiographically negative subarachnoid hemorrhage. AJR Am J Roentgenol 172 : 201-206, 1999

25. Schievink WI, Wijdicks EF : Origin of pretruncal nonaneurysmal subarachnoid hemorrhage: ruptured vein, perforating artery, or intramural hematoma? Mayo Clin Proc 75 : 1169-1173, 2000

26. Tan HW, Chen X, Maingard J, Barras CD, Logan C, Thijs V, et al. : Intracranial vessel wall imaging with magnetic resonance imaging: current techniques and applications. World Neurosurg 112 : 186-198, 2018

27. Woodfield J, Rane N, Cudlip S, Byrne JV : Value of delayed MRI in angiogram-negative subarachnoid haemorrhage. Clin Radiol 69 : 350 356, 2014

28. Yu DW, Jung YJ, Choi BY, Chang CH : Subarachnoid hemorrhage with negative baseline digital subtraction angiography: is repeat digital subtraction angiography necessary? J Cerebrovasc Endovasc Neurosurg $14: 210-215,2012$ 